\title{
Objective Indicators of Health and Function
}

\section{Hugh Nolan, Louise Newman and Orna Donoghue}

\section{Contents}

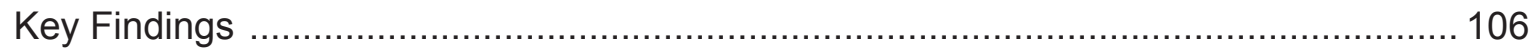

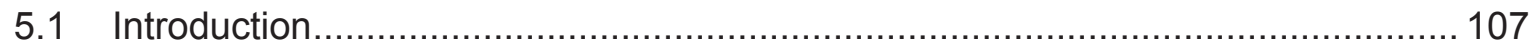

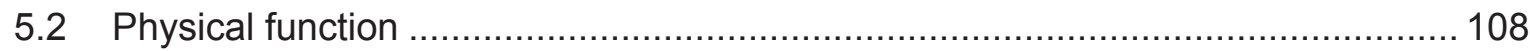

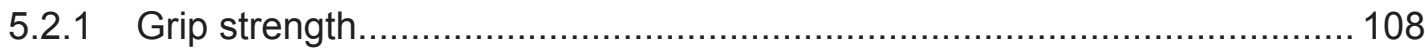

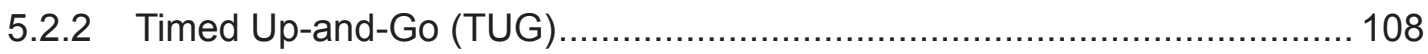

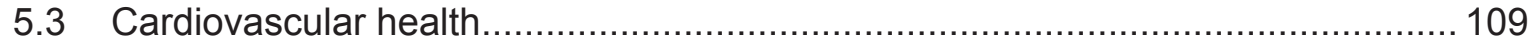

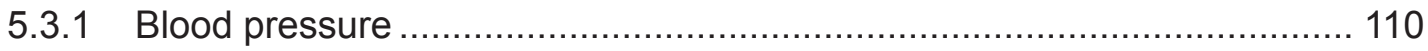

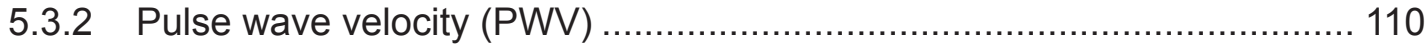

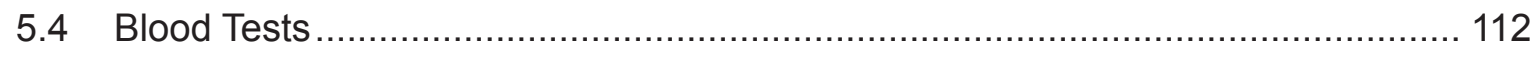

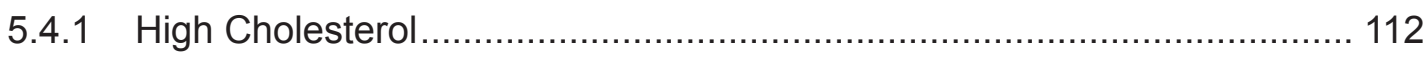

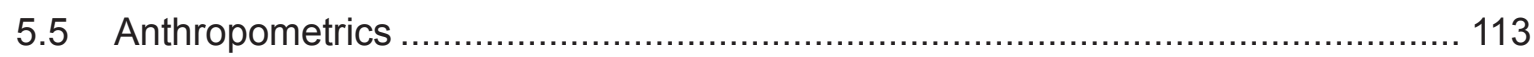

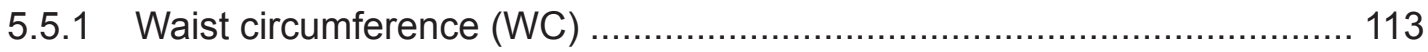

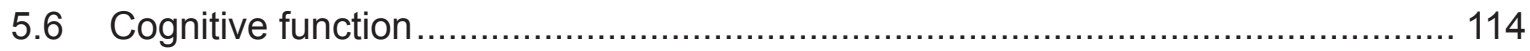

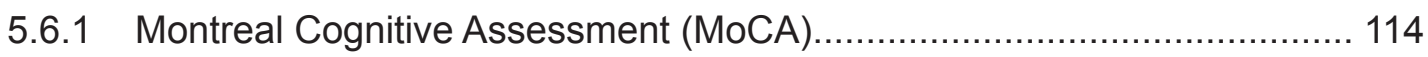

5.6.2 Choice Reaction Time .......................................................................... 115

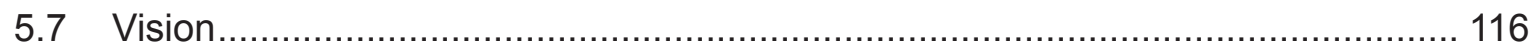

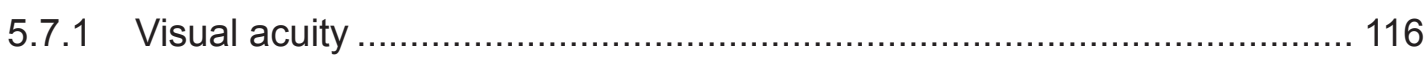

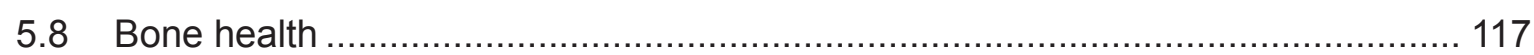

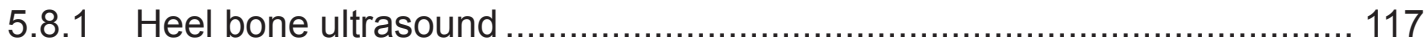

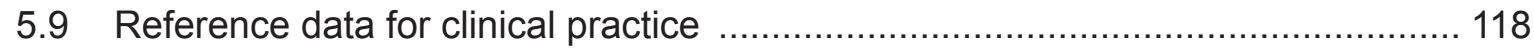

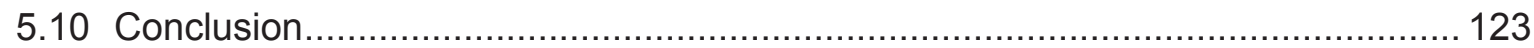

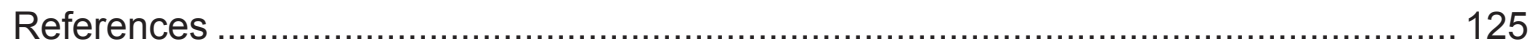




\section{Objective Indicators of Health and Function}

\section{Key Findings}

- The health of community-dwelling older adults in Ireland measured through objective indicators of health and function has remained stable over four years.

- Objective evidence of increased cardiovascular risk is common in older adults; 2 in 5 adults aged 50 years and over had high blood pressure at Wave 1 and this remained high four years later.

- The proportion of adults with high cholesterol declined from 1 in 2 older adults at Wave $1(51 \%)$ to 2 in 5 at Wave $3(41 \%)$; this decline is particularly evident in adults aged less than 75 years.

- A higher proportion of older adults had a substantially increased waist circumference after four years, particularly women aged $50-64$ years (45\% at Wave 1 versus $52 \%$ at Wave 3 ), highlighting the growing problem of obesity.

- Two in five older adults (42\%) reported some level of visual loss at Wave 1 and this increased to over half $(52 \%)$ at Wave 3.

- Reference data for men and women at different ages have been generated for a number of physical and cognitive measures using TILDA data. These can be used in the routine clinical assessment of community-dwelling older adults, for example during the comprehensive geriatric assessment, allowing their performance to be compared to other adults of the same age and gender. 


\subsection{Introduction}

One of the novel components of TILDA is the inclusion of a comprehensive health assessment offered at every second wave, to all participants who complete an interview in that wave. This assessment includes tests of cardiovascular function, cognitive function, mobility, vision, bone health, anthropometry and collection of blood samples.

The information obtained from objective tests is often very different to information obtained from subjective reports and can help identify previously undiagnosed illness. Previous TILDA reports have compared health assessment data to the information provided by participants during their interviews and found that many older adults in Ireland are unaware that they have atrial fibrillation (1), diabetes (2), high blood pressure (3), osteopenia and osteoporosis (4) and clinically relevant depressive symptoms (5). Some objective tests can also give an early indication of decline in health or function prior to symptomatic disease. These biomarkers of ageing can therefore help identify someone with a high risk of developing a specific illness allowing early intervention at a time when it may be most effective for preventing or reducing illness (6).

At Wave 1, TILDA health assessments were conducted by trained research nurses in health centres in Dublin and Cork, and participants who were unable or unwilling to attend a health centre were offered a modified, home-based assessment, also conducted by a research nurse. At Wave 1, 6,150 participants completed a health assessment representing $72 \%$ of the overall TILDA sample. The majority $(86 \%)$ attended a health centre while the remaining 14\% completed a home-based assessment. At Wave 3, 5,391 participants completed a health assessment representing $81 \%$ of those who completed a Wave 3 interview, with an increased proportion (20\%) selecting a home-based assessment in comparison to Wave 1.

This chapter presents objective indicators of health and function for adults aged 50 years and over, who completed the TILDA health assessment at both Wave 1 and Wave 3 $(n=5,045)$, and shows the change in health and function over four years. TILDA measures indicators of health and function in the following domains: physical function, cardiovascular function, cognitive function, bone health, vision, and anthropometrics. The analysis is stratified by age group with participants' ages reflecting their age at Wave 1 . Wave 1 data is then used to illustrate the age-related changes in physical and cognitive function providing reference data or "normative data" that can be used in clinical practice, including in the comprehensive geriatric assessment (CGA). 


\subsection{Physical function}

\subsubsection{Grip strength}

Grip strength is often used as a proxy for overall body strength and research has shown that lower grip strength is associated with an increased risk of future fractures, cognitive decline and mortality $(7,8)$. In TILDA, participants were asked to squeeze a handheld Baseline $\AA$ dynamometer as hard as possible for a few seconds. Grip strength is indicated by the average of two measurements from the dominant hand. Figure 5.1 shows that grip strength is lower in women and declines with increasing age in both men and women. While grip strength remained the same for the majority of older adults between Wave 1 and Wave 3, women aged 50-64 years demonstrated an increase in grip strength at Wave 3 (20.9 $\mathrm{kg}$ at Wave 1 versus $22.1 \mathrm{~kg}$ at Wave 3$)$.

Figure 5.1: Grip strength at Waves 1 and 3, by age group and sex

\begin{tabular}{|l}
$\square$ Wave $1 \quad$ Wave 3 \\
\hline
\end{tabular}

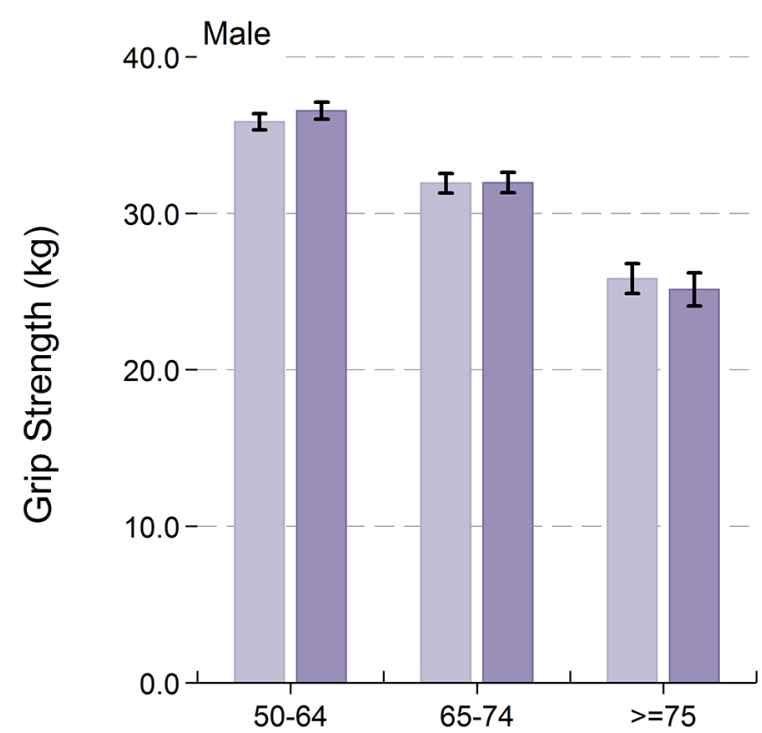

Female

Note. $\mathrm{N}=3909$; Missing obs $=1136$; Error bars correspond to $95 \%$ confidence intervals

\subsubsection{Timed Up-and-Go (TUG)}

Timed Up-and-Go (TUG) is a simple mobility test (9) that is often used in clinical practice to assess an individual's risk of having a fall. Participants were asked to stand up from a chair, walk 3 metres, turn around, walk back to the chair and sit down again. The time 
taken to complete this task was measured using a stopwatch. Figure 5.2 shows TUG performance for older adults in Ireland, stratified by age group and sex. There is a clear age-related gradient with slower performance in older adults. The time taken to complete TUG at Wave 3 increased in both men and women and across all age groups, reflecting worse mobility at Wave 3 compared to Wave 1 . This increase in time taken to complete TUG is most pronounced in men aged 75 years and over (12.1 s versus $14.5 \mathrm{~s}$ ) and women aged 75 years and over (12.1s versus $15.6 \mathrm{~s})$.

Figure 5.2: Timed Up-and-Go (TUG) performance at Waves 1 and 3, by age group and sex 20.0 - Male $^{-}$

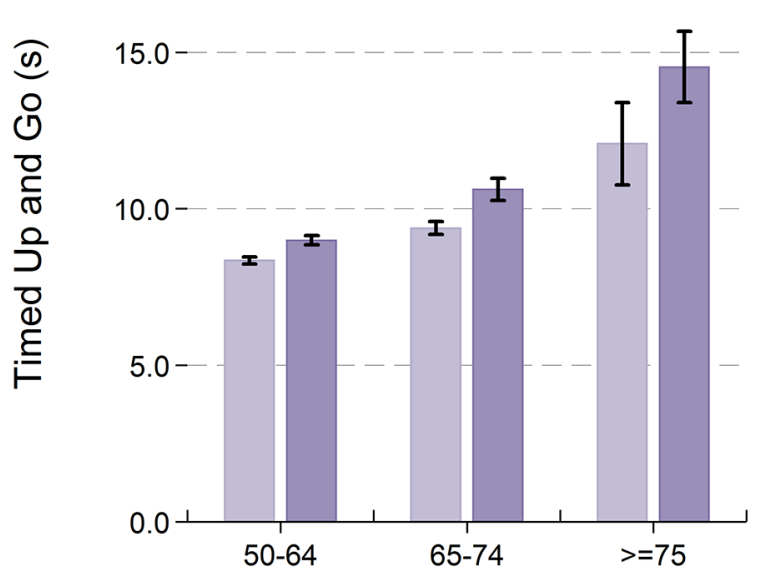

Female

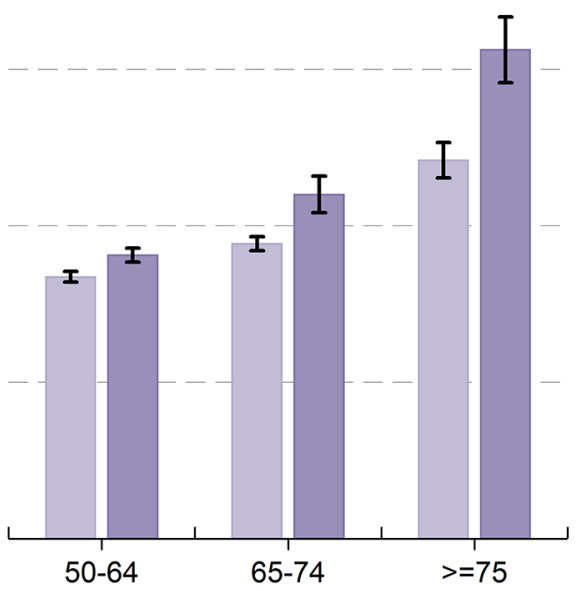

Note. $\mathrm{N}=4251 ;$ Missing obs $=794 ;$ Error bars correspond to $95 \%$ confidence intervals

\subsection{Cardiovascular health}

Cardiovascular health can be measured in a number of ways. Blood pressure is a very good indicator of cardiovascular health and represents a modifiable risk factor for adverse cardiovascular events such as coronary heart disease and stroke (10). Pulse wave velocity (PWV) is the speed with which blood moves through the arteries. A higher PWV is an indicator of increased arterial stiffness and a sign of atherosclerosis. Arterial stiffness increases with age in most populations, and has been shown to independently predict adverse cardiovascular events (11). As it is closely linked with blood pressure, it can similarly be improved with lifestyle changes and medication. 


\subsubsection{Blood pressure}

The European Society of Cardiology 2007 Guidelines for Cardiovascular Disease Prevention classifies normal systolic blood pressure (SBP) and diastolic blood pressure (DBP) as $<140 \mathrm{mmHg}$ and $<90 \mathrm{mmHg}$ respectively. High blood pressure (hypertension) is defined as SBP $\geq 140 \mathrm{mmHg}$ or DBP $\geq 90 \mathrm{mmHg}$ (12). In TILDA, mean blood pressure was calculated from two seated readings obtained 1 minute apart using an Omron ${ }^{\mathrm{TM}}$ digital oscillometric blood pressure monitor. The proportion of older adults with evidence of hypertension was high and remained consistent after 4 years ( $44 \%$ at Wave 1 versus $40 \%$ at Wave 3), with no differences by age group or sex. However, these proportions mask change at the individual level where $16 \%$ of those with hypertension at Wave $1 \mathrm{did}$ not have hypertension at Wave 3 and conversely, 13\% of those with normal blood pressure at Wave 1 displayed hypertension at Wave 3. This may have been influenced by feedback about blood pressure given to participants and their GPs (with their permission) after the health assessment.

\subsubsection{Pulse wave velocity (PWV)}

In TILDA, the average of two PWV measurements between the carotid and femoral arteries was obtained using a Vicorder $®$, which is the gold standard non-invasive method of measuring arterial stiffness. The European Society of Hypertension and the European Society of Cardiology suggest that PWV of $>12 \mathrm{~m} / \mathrm{s}$ is indicative of asymptomatic organ damage affecting the heart, brain, kidney, eye or peripheral arteries (10). Figure 5.3 shows an age-related increase in PWV, and higher PWV in men compared to women in all age groups. Two thirds of men and over half of women aged 75 years and older have asymptomatic organ damage based on the criteria above. The proportion of older adults with high PWV did not change between Wave 1 and Wave 3. 
Figure 5.3: Proportion (\%) of older adults in Ireland with a pulse wave velocity (PWV) $>12$ $\mathrm{m} / \mathrm{s}$ at Waves 1 and 3, by age group and sex

\section{Wave $1 \square$ Wave 3}

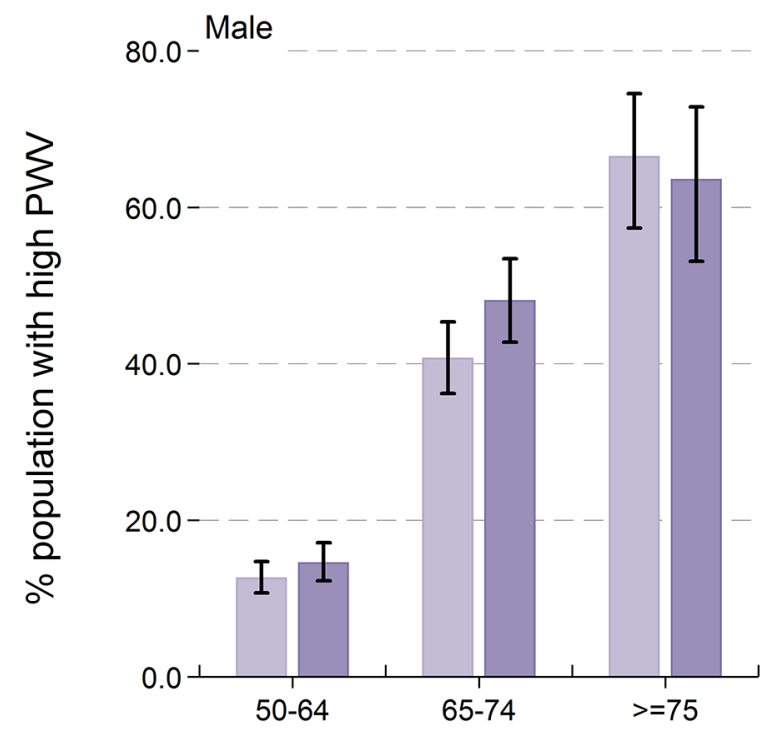

Female

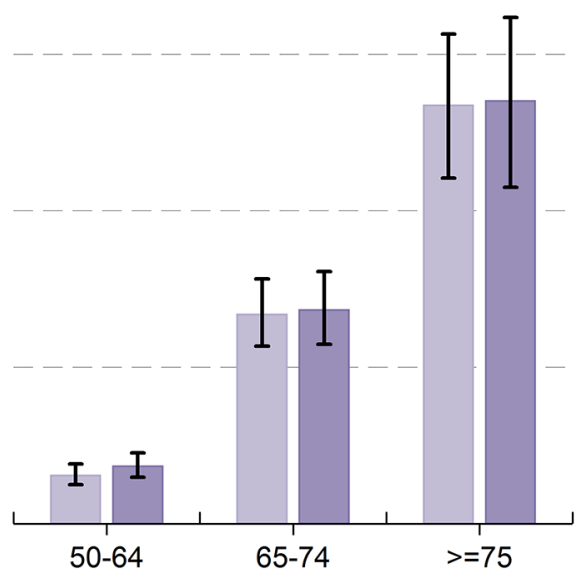

Note. $\mathrm{N}=3223$; Missing obs $=1822$; Error bars correspond to $95 \%$ confidence intervals 


\subsection{Blood Tests}

\subsubsection{High Cholesterol}

Total serum cholesterol is a measure of the amount of lipoproteins in the bloodstream, measured from a blood sample. This is of low-density lipids (LDLs), high-density lipids (HDLs) and triglycerides. It is important to maintain appropriate levels of cholesterol as a build-up in the arteries can lead to atherosclerosis, impaired blood flow, clots and possible heart attack. Total serum cholesterol levels above $5 \mathrm{mMol} / \mathrm{L}$ are considered higher than optimal and associated with an increased risk of adverse cardiovascular events (13). Overall, $41 \%$ of older adults in Ireland have cholesterol levels above $5 \mathrm{mMol} / \mathrm{L}$ (men: $31 \%$, women: $51 \%$ ) at Wave 3 , and this is a reduction from Wave $1(51 \%)$. Figure 5.4 illustrates that this reduction is evident in those aged less than 75 years, but most notably in men aged $65-74$ years (31\% at Wave 1 to $17 \%$ at Wave 3 ). The proportion with high cholesterol also declines with increasing age but is higher in women than men at all ages.

Figure 5.4: Proportion (\%) of older adults in Ireland with total serum cholesterol level above $5.0 \mathrm{mMol} / \mathrm{L}$ at Waves 1 and 3, by age group and sex

Wave $1 \square$ Wave 3

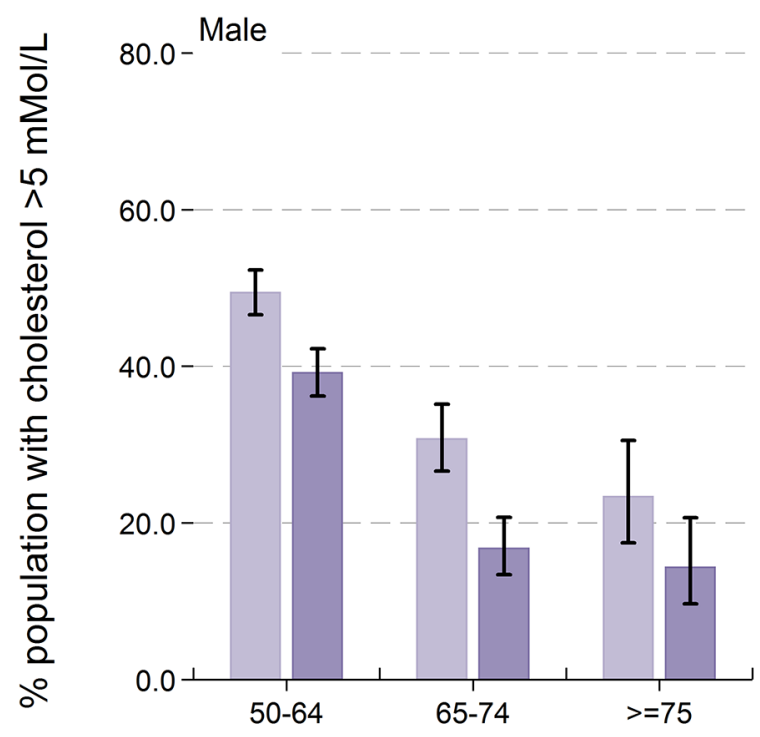

Female

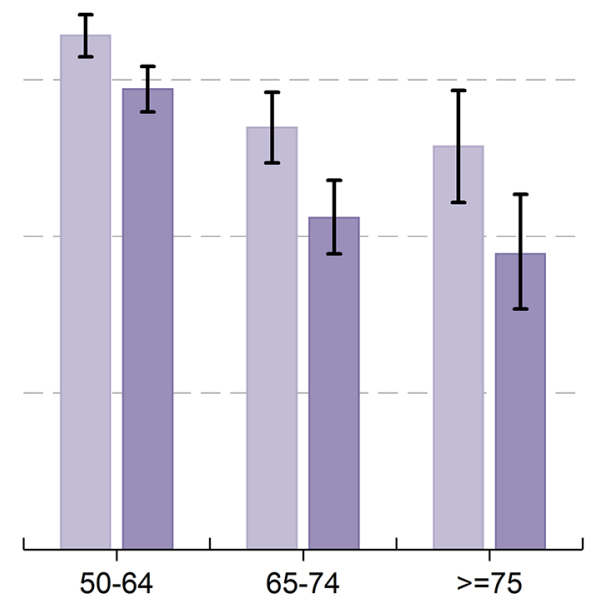

Note. $\mathrm{N}=3978$; Missing obs $=1067$; Error bars correspond to $95 \%$ confidence intervals 


\subsection{Anthropometrics}

\subsubsection{Waist circumference (WC)}

Obesity is commonly defined according to body mass index (weight/height ${ }^{2}$ ), however this measure is not as useful in older adults as it does not reflect the loss of muscle mass or increase in abdominal fat mass that are commonly observed with increasing age (14). Waist circumference (WC) is considered a more useful measure of excess fat in older adults as it is a measure of visceral fat which has been linked to metabolic disturbances, increased risk for cardiovascular disease, type 2 diabetes and increased risk of breast cancer. The World Health Organisation suggest the following definitions should be applied for WC: men - normal $<94 \mathrm{~cm}$, increased $94-101 \mathrm{~cm}$, substantially increased $\geq 102 \mathrm{~cm}$; women - normal $<80 \mathrm{~cm}$, increased $80-87 \mathrm{~cm}$, substantially increased $\geq 88 \mathrm{~cm}$.

Approximately half of older adults in Ireland have a substantially increased WC and this is higher in women than men (Figure 5.5). Between Wave 1 and Wave 3, the proportion with a normal WC decreased from $24 \%$ to $21 \%$ while the proportion with substantially increased WC increased from $49 \%$ to $54 \%$. This change was particularly evident in women aged $50-$ 64 years where $19 \%$ had a normal and $57 \%$ had a substantially increased WC at Wave 3 , compared to $25 \%$ (normal WC) and 49\% (substantially increased WC) at Wave 1.

Figure 5.5: Proportion (\%) of older adults in Ireland with normal, increased or substantially increased waist circumference at Waves 1 and 3, by age group and sex

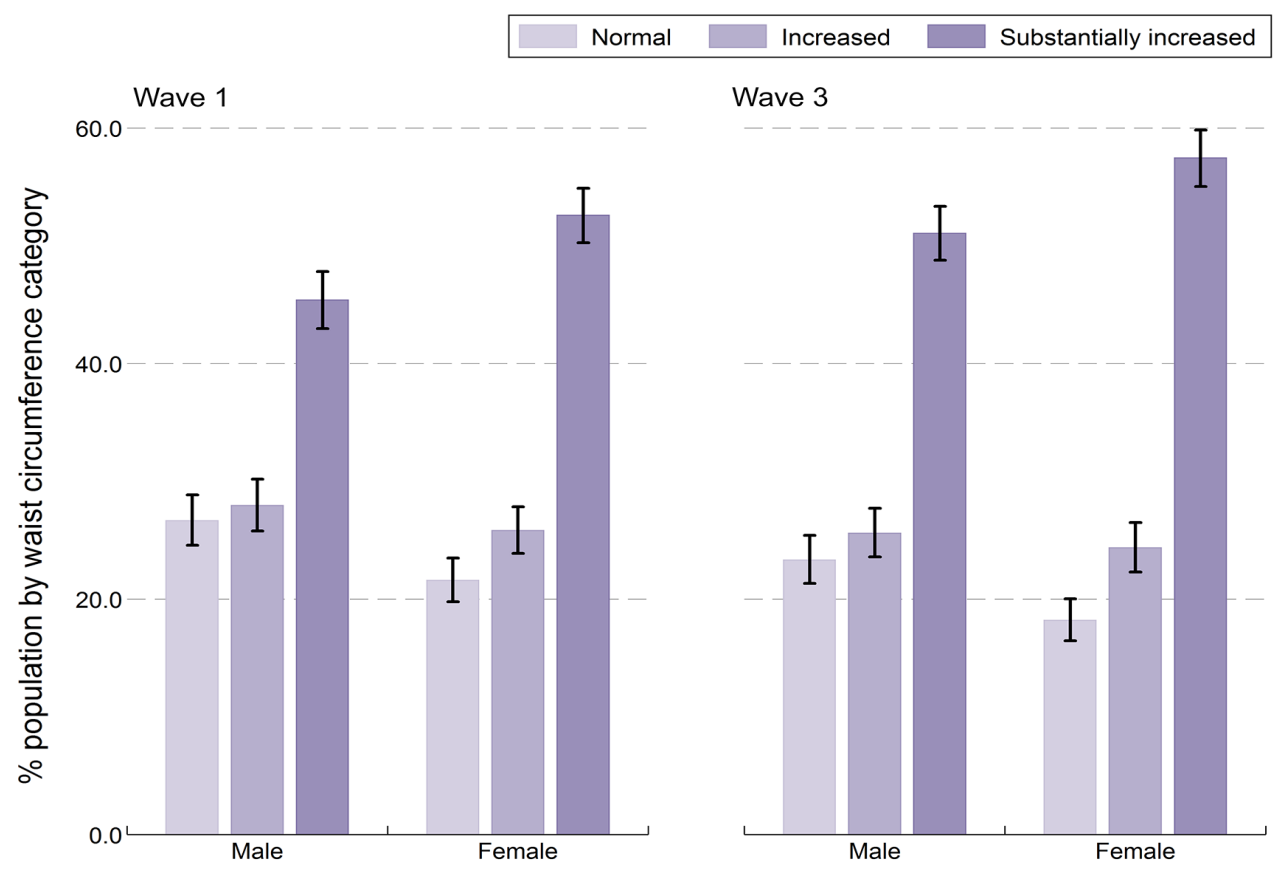

Note. $\mathrm{N}=4404$; Missing obs $=0$; Error bars correspond to $95 \%$ confidence intervals 


\subsection{Cognitive function}

TILDA measures function in a number of cognitive domains. For the purposes of this report, we describe changes in global cognitive function using the Montreal Cognitive Assessment (MoCA), and speed of reaction, measured by the Choice Reaction Time test.

\subsubsection{Montreal Cognitive Assessment (MoCA)}

MoCA is a test of global cognitive ability and assesses function across multiple domains of cognition including memory recall, visuospatial ability, executive function, attention, language, and orientation to time/place. The test is frequently used in clinical practice and has a maximum score of 30 . Figure 5.6 shows that global cognitive function declines with increasing age and is similar in men and women. There was little change in cognitive function between Wave 1 and Wave 3 with the exception of a small improvement in women aged 50-64 years (25.5 at Wave 1 versus 26.0 at Wave 3 ) and a decline in women aged 75 years and older (22.1 at Wave 1 versus 20.6 at Wave 3 ).

Figure 5.6: Montreal Cognitive Assessment (MoCA) score at Waves 1 and 3, by age group and sex
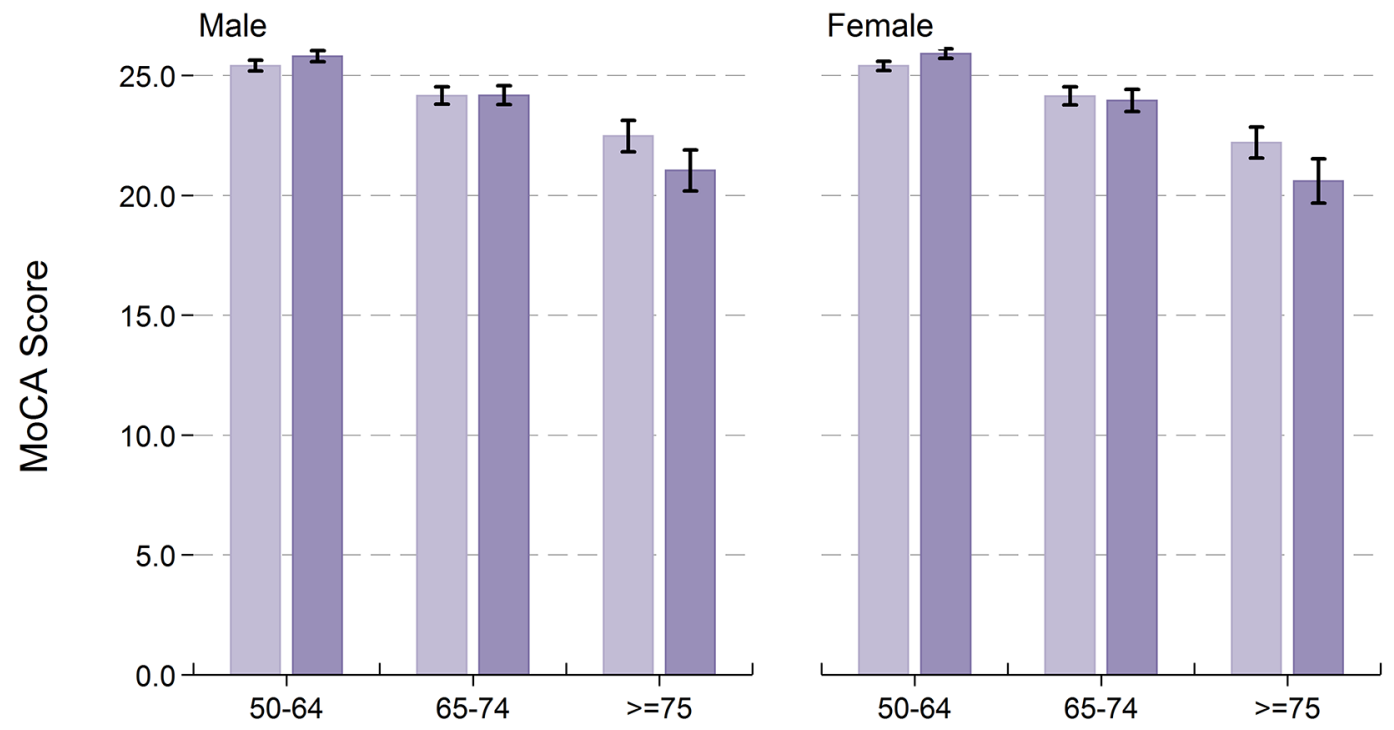

Note. $\mathrm{N}=4397$; Missing obs $=648$; Error bars correspond to $95 \%$ confidence intervals 


\subsubsection{Choice Reaction Time}

The Choice Reaction Time test is a computerised test which measures attention and speed of processing information in the brain. Participants pressed a button on the keyboard until an on-screen stimulus prompted them to press another pre-specified button. Average reaction time from presentation of the stimulus to pressing the button, was measured over multiple trials. Processing speed is important as it is thought to underlie all aspects of cognitive function while attention is important for many everyday activities (15). Figure 5.7 shows an increase in choice reaction time, indicating slower processing and movement speed with increasing age in both men and women. There is also evidence of slower reaction time at Wave 3 compared to Wave 1 in men aged 65-74 years (a 12\% decline from 858 milliseconds (ms) at Wave 1 to $957 \mathrm{~ms}$ at Wave 3 ) and women aged 50-64 years (an $8 \%$ decline from $787 \mathrm{~ms}$ at Wave 1 to $850 \mathrm{~ms}$ at Wave 3 ).

Figure 5.7: Choice reaction time at Waves 1 and 3, by age group and sex

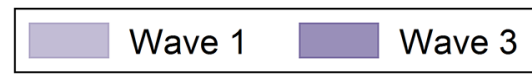
$1500.0-$ Male

Female
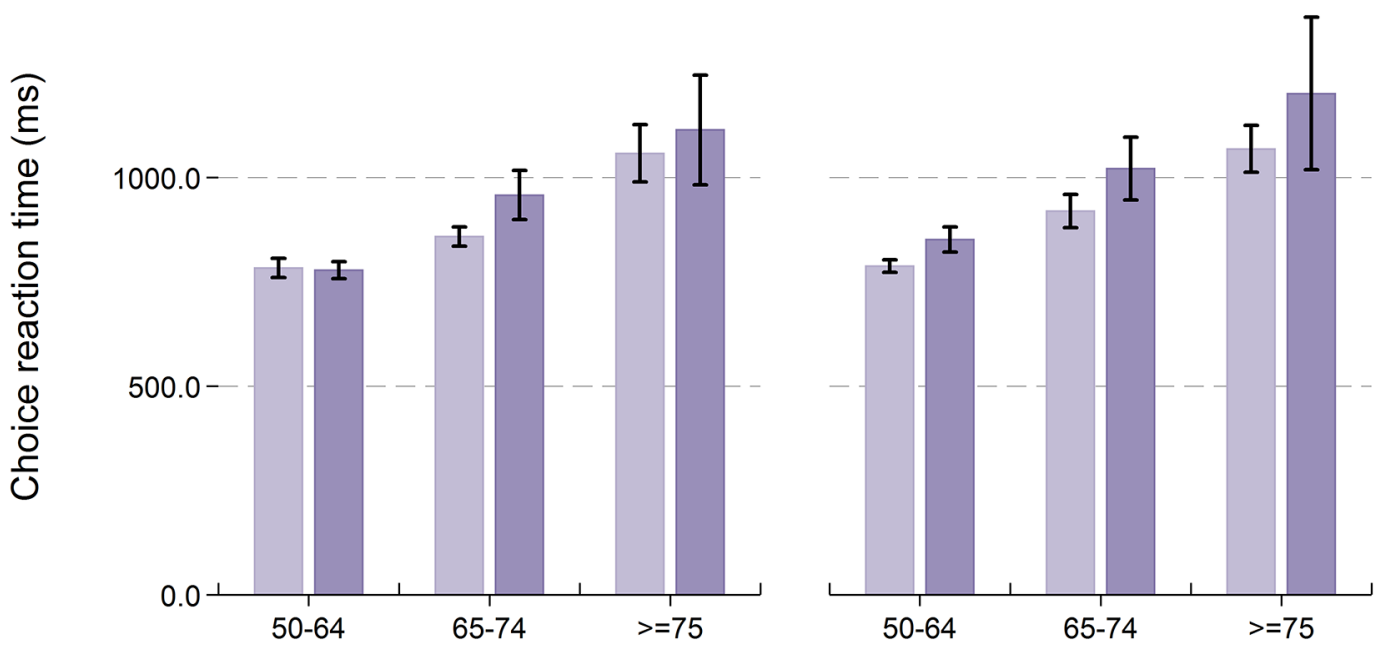

Note. $\mathrm{N}=3520$; Missing obs $=1525$; Error bars correspond to $95 \%$ confidence intervals 


\subsection{Vision}

\subsubsection{Visual acuity}

Visual acuity is the clarity of vision and is assessed using a LogMAR (Minimal Angle of Resolution) chart. It reflects the ability to detect progressively smaller, high contrast, black letters on a white background, and is commonly used by GPs and opticians. The score for the best eye was used in this analysis and as participants were allowed to wear glasses/ lenses for this test, measurements reflect corrected visual acuity. The World Health Organisation categories of visual impairment define mild vision loss as a LogMAR score of $\geq 0.2$, with more pronounced levels of visual loss (moderate, severe or blind) defined as scores $\geq 0.6(16)$. Impaired vision is a modifiable risk factor for accidents and falls. The prevalence of moderate or severe visual loss or blindness in older adults is low at $2.6 \%$, however mild visual loss is very common, even for those using corrective lenses (36\%). Figure 5.8 shows that the proportion of people with any vision loss increases with age. The proportion of adults aged 50-64 years with visual loss increases from $37 \%$ at Wave 1 to $46 \%$ at Wave 3 and is higher in women than men (men: $33 \%$ at Wave 1 versus $43 \%$ at Wave 3; women: $41 \%$ at Wave 1 versus $49 \%$ at Wave 3 ). The prevalence of visual loss also increased in women aged $65-74$ years over four years (53\% at Wave 1 versus $63 \%$ at Wave 3).

Figure 5.8: Proportion (\%) of older adults in Ireland with any visual loss (mild, moderate, severe or blind) at Waves 1 and 3, by age group and sex

Wave $1 \square$ Wave 3 $100.0^{-}$Male

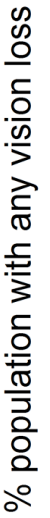

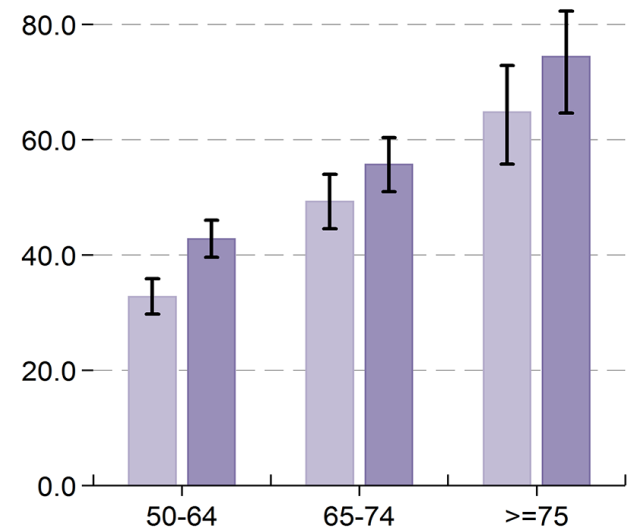

Female

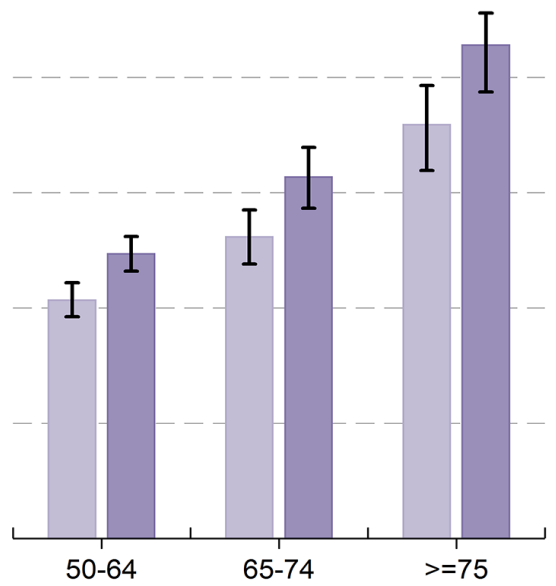

Note. $\mathrm{N}=3568$; Missing obs $=1477$; Error bars correspond to $95 \%$ confidence intervals 


\subsection{Bone health}

\subsubsection{Heel bone ultrasound}

Fractures, particularly hip fractures, have debilitating consequences for older adults and place significant demands on the healthcare services. In TILDA, risk of osteoporotic fracture was assessed using quantitative ultrasound of the calcaneus or heel bone (Achilles Heel Ultrasound, Lunar, Madison, USA). This device measures the broadband ultrasound attenuation (BUA) and the speed of sound (SOS), both of which provide an index of bone stiffness. This stiffness index can predict fracture risk better than either BUA or SOS alone (17), however it should be noted that it is not diagnostic. An individual is considered to have osteoporosis, osteopenia or normal bone density if the stiffness index is $\leq 65 \%, 66-86 \%$, or $>86 \%$ respectively (18). Over half of women and over onequarter of men aged 54 years and older show evidence of osteopenia in Wave 3 (Figure 5.9). Osteoporosis affects three times as many women as men and the prevalence increases with age in women. At Wave 1,7\% of women aged 50-64 years had evidence of osteoporosis compared to $15 \%$ of those aged $65-74$ years and $30 \%$ of those aged 75 years and older.

Figure 5.9: Proportion (\%) of older adults in Ireland with osteoporosis and osteopenia at Waves 1 and 3, by sex

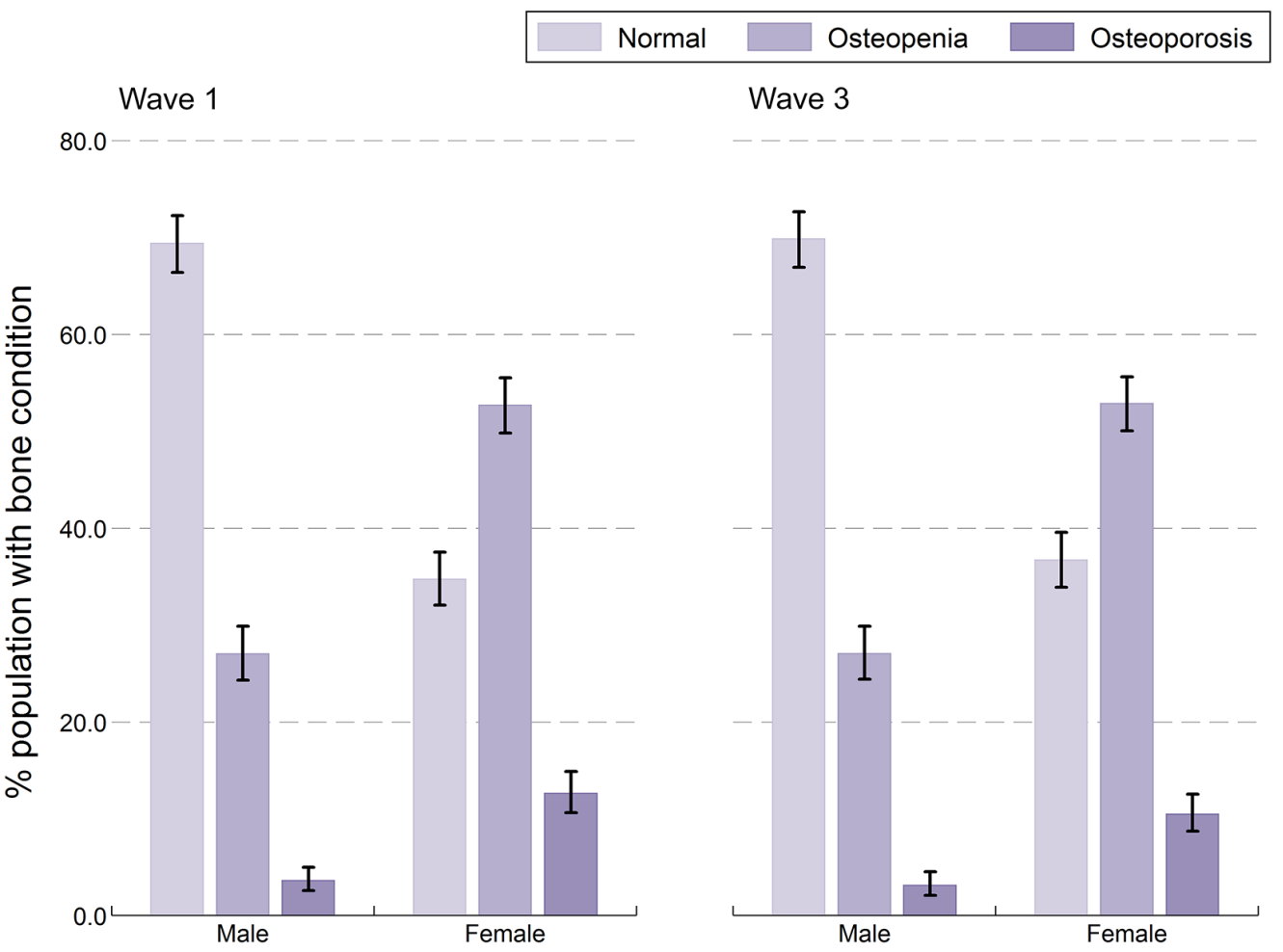

Note. $\mathrm{N}=2754$; Missing obs $=0$; Error bars correspond to $95 \%$ confidence intervals 


\subsection{Reference data for clinical practice}

It is well accepted and clearly shown in this chapter that physical and cognitive function varies with age and sex. Therefore, it is important to consider age, sex and any other relevant variables when reviewing an individual's performance on a particular test of physical and cognitive function. Normative data allows a researcher and a clinician to judge an individual's performance on a test relative to others of similar age, sex, educational level, height, etc. and identify a need for further monitoring and/or intervention. Normative data are often generated from standardised tests or measures obtained in epidemiological studies and designed for use and interpretation in clinical and research settings. For example, height and weight charts are routinely used for babies and children to assess if they are growing within optimal ranges.

Based on Wave 1 TILDA data, normative data tables and graphs were published for a range of measures including height, weight, normal walking speed, TUG, grip strength, cognitive tests (Mini-Mental State Examination (MMSE), MoCA, Colour Trails test), bone mineral density and beat-to-beat orthostatic blood pressure measurements $(19,20)$. The advantages of generating normative data from TILDA is that they are based on a large, nationally representative, population-based sample of adults aged 50 years and over who completed a wide range of tests during the health assessment. Prior to this, established national or international normative data did not exist for many of these tests, therefore TILDA was able to generate these for the first time. The detailed statistical modelling techniques that were used to generate the data mean that the results are robust $(19,20)$.

Normative data are typically provided in graphical and/or tabular format and show how an individual performs compared to similar individuals in the population. Depending on the test, these data may be stratified by sex, educational level, height or any other factor that influences performance. Figure 5.10 provides normative values for TUG by age and sex. In general, men walk more quickly than women and height affects how quickly both men and women walk. Therefore, different normative values are provided for men and women within two groups representing taller (men $\geq 173 \mathrm{~cm}$, women $\geq 160 \mathrm{~cm}$ ) and shorter individuals (men $<173 \mathrm{~cm}$, women $<160 \mathrm{~cm}$ ). TUG time increases with age, indicating a slower performance. The difference between men and women is most evident in the older age groups where women perform more slowly than men. 
Figure 5.10: Reference or normative data for Timed Up-and-Go for men and women of different height categories, based on data obtained in TILDA (graphical format) (19)
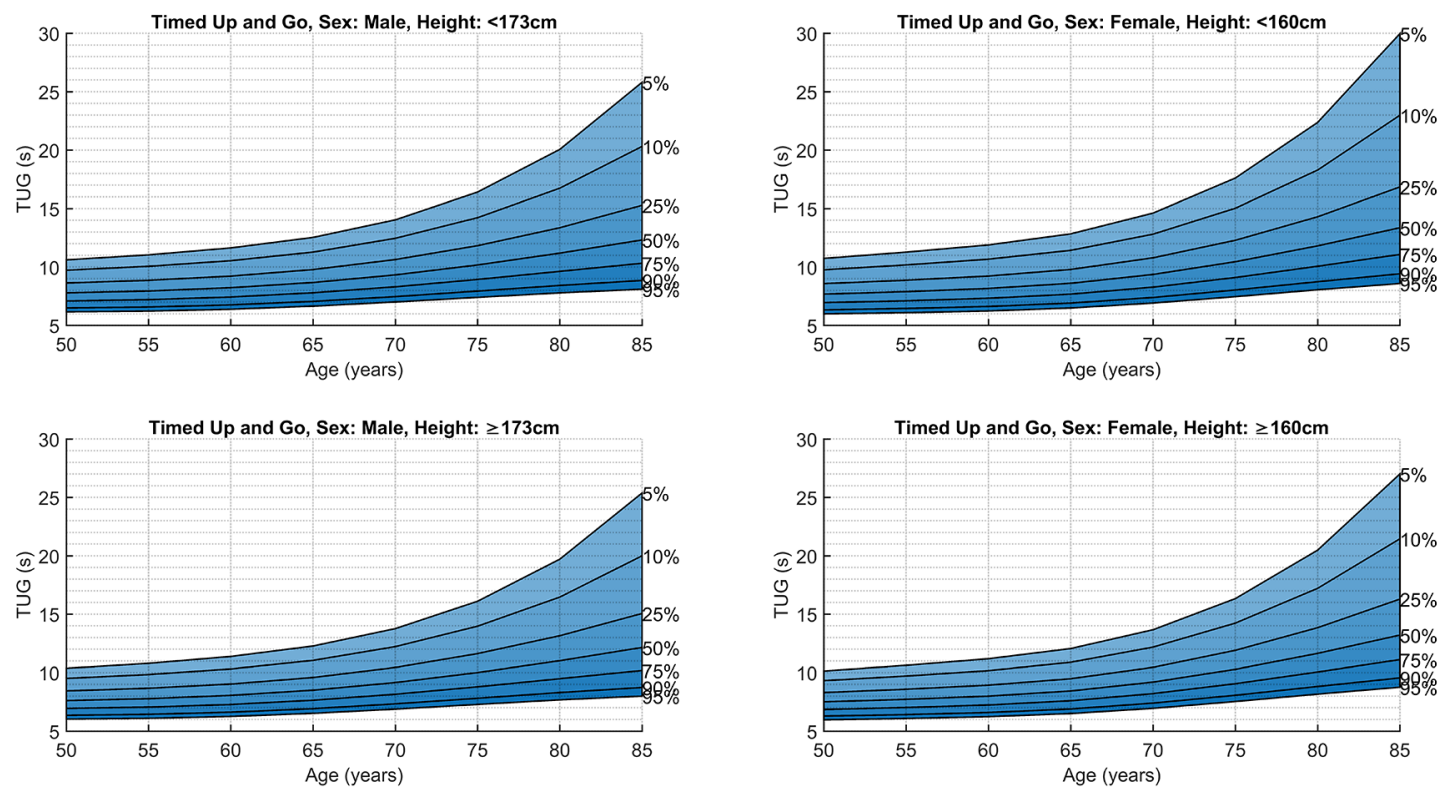

To illustrate how these can be interpreted, a 70 year old woman of height $159 \mathrm{~cm}$ and who performs TUG in 10 seconds is taken as an example. To determine where her performance lies relative to the rest of the population, the reference chart for TUG (women of height $<160 \mathrm{~m}$ ) is consulted (Figure 5.11). A vertical line is drawn from the individual's age on the $x$-axis. A horizontal line is drawn from the performance score on the $y$-axis. The point where these lines meet falls approximately halfway between the 50th and 25th percentile lines, indicating that this individual's performance lies at approximately the 37.5th percentile for a 70 year old woman. Similarly, the percentile can be identified from the corresponding table (Table 5.1). 
Figure 5.11: Interpreting the reference or normative data for Timed Up-and-Go, which is based on data obtained in TILDA (19)

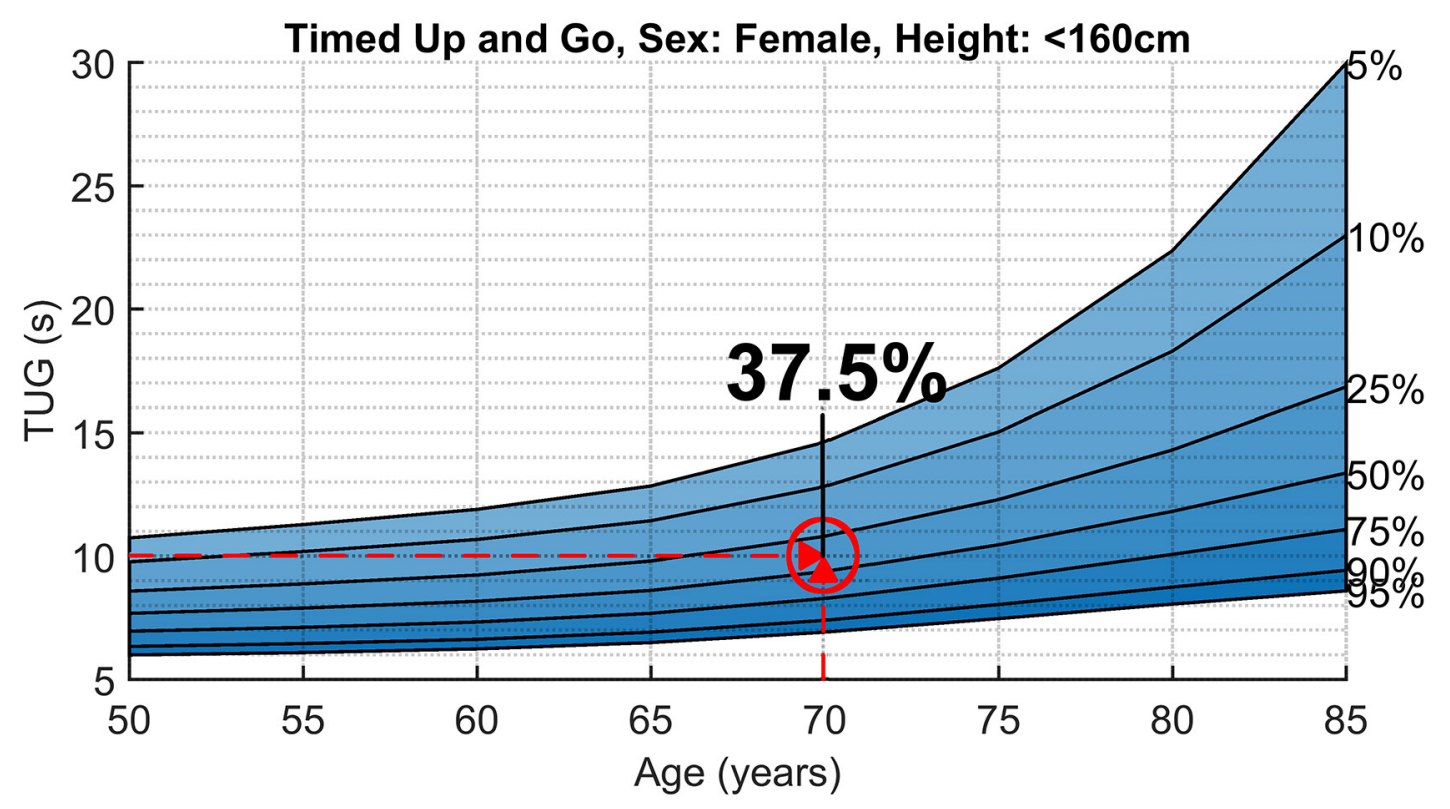


Table 5.1: Reference or normative data for Timed Up-and-Go for men and women of different height categories, based on data obtained in TILDA (tabular format)

\begin{tabular}{|c|c|c|c|c|c|c|c|c|c|}
\hline \multirow{2}{*}{ Sex } & \multirow{2}{*}{ Height } & \multirow{2}{*}{ Age } & \multicolumn{7}{|c|}{ Percentiles of Timed Up-and-Go test scores (seconds) } \\
\hline & & & $95 \%$ & $90 \%$ & $75 \%$ & $50 \%$ & $25 \%$ & $10 \%$ & $5 \%$ \\
\hline \multirow{8}{*}{ Men } & \multirow{8}{*}{$<173 \mathrm{~cm}$} & 50 & 6.2 & 6.5 & 7.1 & 7.8 & 8.6 & 9.7 & 10.6 \\
\hline & & 55 & 6.2 & 6.6 & 7.2 & 7.9 & 8.9 & 10.1 & 11.0 \\
\hline & & 60 & 6.4 & 6.8 & 7.4 & 8.2 & 9.2 & 10.5 & 11.6 \\
\hline & & 65 & 6.6 & 7.1 & 7.8 & 8.7 & 9.8 & 11.3 & 12.5 \\
\hline & & 70 & 7.0 & 7.5 & 8.3 & 9.3 & 10.6 & 12.5 & 14.0 \\
\hline & & 75 & 7.4 & 7.9 & 8.9 & 10.2 & 11.8 & 14.2 & 16.4 \\
\hline & & 80 & 7.8 & 8.4 & 9.6 & 11.2 & 13.4 & 16.7 & 20.1 \\
\hline & & 85 & 8.1 & 8.9 & 10.3 & 12.3 & 15.3 & 20.3 & 25.8 \\
\hline \multirow{8}{*}{ Women } & \multirow{8}{*}{$<160 \mathrm{~cm}$} & 50 & 6.0 & 6.3 & 7.0 & 7.7 & 8.6 & 9.8 & 10.7 \\
\hline & & 55 & 6.1 & 6.5 & 7.1 & 7.9 & 8.9 & 10.2 & 11.3 \\
\hline & & 60 & 6.2 & 6.6 & 7.3 & 8.2 & 9.2 & 10.7 & 11.9 \\
\hline & & 65 & 6.5 & 6.9 & 7.7 & 8.6 & 9.8 & 11.4 & 12.8 \\
\hline & & 70 & 6.9 & 7.4 & 8.3 & 9.4 & 10.8 & 12.8 & 14.6 \\
\hline & & 75 & 7.5 & 8.0 & 9.1 & 10.5 & 12.3 & 15.0 & 17.6 \\
\hline & & 80 & 8.1 & 8.7 & 10.1 & 11.8 & 14.3 & 18.3 & 22.4 \\
\hline & & 85 & 8.6 & 9.4 & 11.1 & 13.4 & 16.8 & 23.0 & 30.0 \\
\hline \multirow{8}{*}{ Men } & \multirow{8}{*}{$\geq 173 \mathrm{~cm}$} & 50 & 6.0 & 6.4 & 6.9 & 7.6 & 8.4 & 9.5 & 10.4 \\
\hline & & 55 & 6.1 & 6.4 & 7.1 & 7.8 & 8.7 & 9.8 & 10.8 \\
\hline & & 60 & 6.3 & 6.6 & 7.3 & 8.1 & 9.0 & 10.3 & 11.4 \\
\hline & & 65 & 6.5 & 6.9 & 7.6 & 8.5 & 9.6 & 11.0 & 12.3 \\
\hline & & 70 & 6.9 & 7.3 & 8.2 & 9.2 & 10.4 & 12.2 & 13.8 \\
\hline & & 75 & 7.3 & 7.8 & 8.8 & 10.0 & 11.6 & 14.0 & 16.1 \\
\hline & & 80 & 7.7 & 8.3 & 9.5 & 11.0 & 13.2 & 16.5 & 19.7 \\
\hline & & 85 & 8.0 & 8.7 & 10.2 & 12.1 & 15.1 & 20.0 & 25.4 \\
\hline \multirow{8}{*}{ Women } & \multirow{8}{*}{$\geq 160 \mathrm{~cm}$} & 50 & 6.0 & 6.3 & 6.9 & 7.5 & 8.3 & 9.3 & 10.1 \\
\hline & & 55 & 6.1 & 6.4 & 7.0 & 7.7 & 8.6 & 9.7 & 10.6 \\
\hline & & 60 & 6.2 & 6.6 & 7.2 & 8.0 & 8.9 & 10.2 & 11.2 \\
\hline & & 65 & 6.5 & 6.9 & 7.6 & 8.4 & 9.5 & 10.9 & 12.1 \\
\hline & & 70 & 6.9 & 7.4 & 8.2 & 9.2 & 10.5 & 12.2 & 13.7 \\
\hline & & 75 & 7.5 & 8.1 & 9.1 & 10.3 & 11.9 & 14.2 & 16.3 \\
\hline & & 80 & 8.2 & 8.8 & 10.1 & 11.6 & 13.8 & 17.2 & 20.5 \\
\hline & & 85 & 8.8 & 9.6 & 11.1 & 13.2 & 16.3 & 21.5 & 27.0 \\
\hline
\end{tabular}


Computer programmes and applications on tablets (iPad, Android, etc) where individual details (e.g. age, sex, height) and performance score is entered allows an even more precise estimate of performance to be obtained (Figure 5.12). The Department of Medical Physics in St. James's Hospital, Dublin, have developed such an app in conjunction with TILDA for iPhone/iPad. Clinicians can use this information to identify sub-optimal performance, to determine if an intervention is required and to monitor change as a result of an intervention.

Figure 5.12: Tablet-based application allowing comparison of an individual test performance to normative data

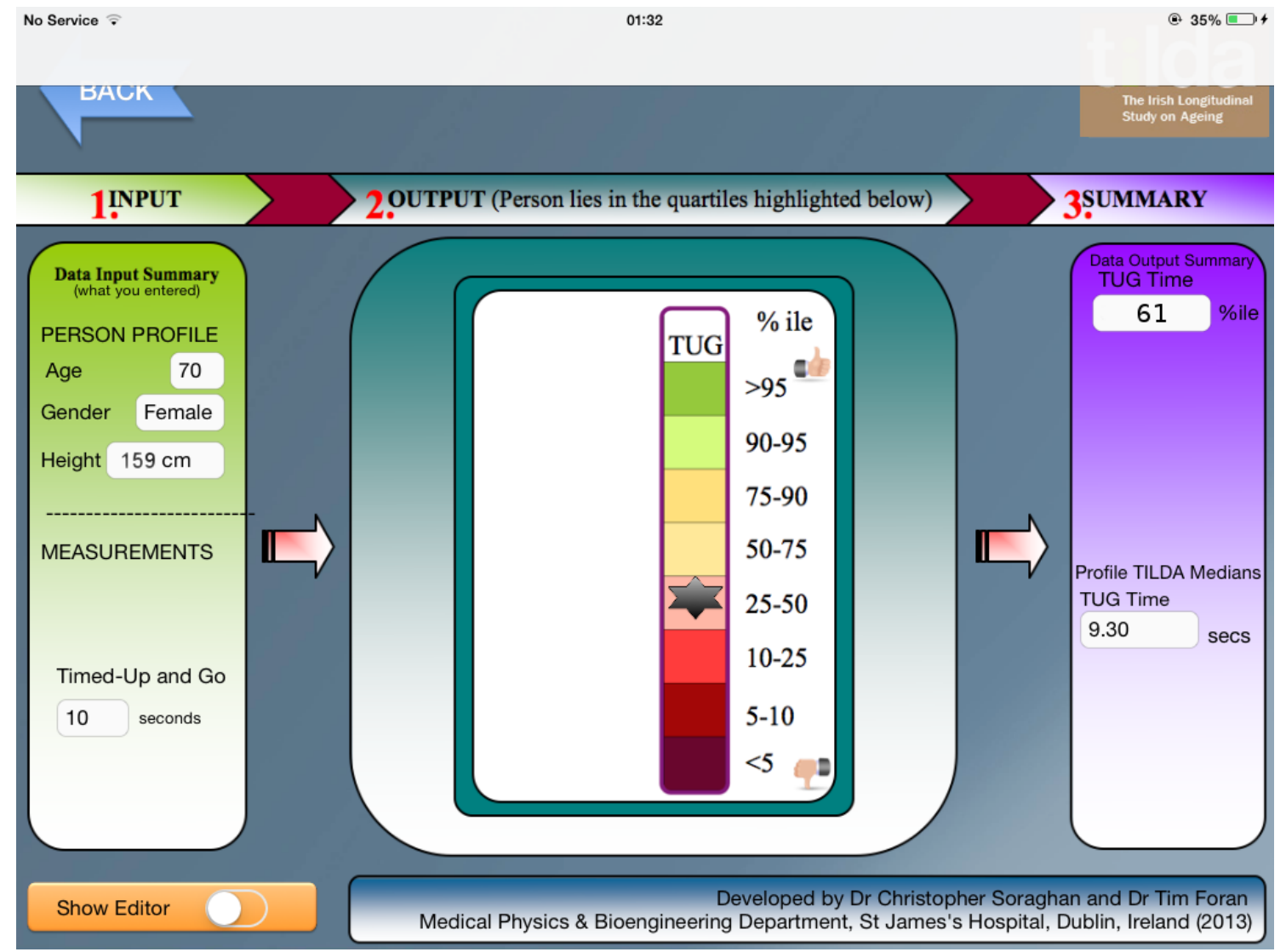




\subsection{Conclusion}

These results illustrate the change in objective indicators of health and function in community-dwelling older adults in Ireland over four years. In general, there were limited changes between Waves 1 and 3 indicating stable health. This is not altogether unexpected as TILDA participants recruited at baseline were relatively healthy and four years is a short follow-up period. However, there were some small differences which have notable implications for health.

Visual loss which can affect independence and quality of life, became more prevalent, particularly in adults aged less than 75 years. An increasing proportion of Irish men and women aged 50-64 years at Wave 1 also had substantially increased WC four years later. This highlights the growing problem of obesity in older adults in Ireland, which has already been documented $(2,21)$. Obesity is associated with several negative health outcomes such as increased risk of metabolic and cardiovascular disease, musculoskeletal problems, decreased physical function, and some cancers (14).

This negative effect on health is compounded by the high proportion of older adults in Ireland who showed evidence of high blood pressure and high PWV, which is indicative of asymptomatic organ damage. It is also consistent with low levels of physical activity previously reported in this group (22). However, on a more positive note, the proportion of adults with high cholesterol, particularly those aged less than 75 years, declined at Wave 3 compared to Wave 1. As participants and their GPs (if they gave permission for this) were informed if they had high cholesterol at Wave 1, it is possible that this decline might have been a consequence of subsequent monitoring and management either through medications or through recommended changes in diet and exercise.

Normative data presented here provide a valuable resource that is suitable for use in the clinical assessment of community-dwelling older adults in Ireland. In particular, these data can play an integral role in comprehensive geriatric assessment (CGA) which reviews an older person's medical conditions, mental health, functional capacity and social circumstances with the intention of developing and implementing an integrated assessment, intervention and review of an older person's individual needs (23). CGA is often conducted by a multidisciplinary team and while there are recommended domains that should be assessed, these are often determined by local practice, expertise and setting. Generally, it involves a number of objective assessments, for example, of mobility and cognition. The availability of evidence-based, reliable and up-to-date normative data is very important to allow clinicians to correctly compare and interpret test scores relative 
to the general population. Poor scores in many of these objective measures e.g. TUG and grip strength, are associated with an increased risk of outcomes such as disability, cognitive decline, hospitalisation and mortality $(7,8)$. Early detection of decline in health or function, particularly when an individual is asymptomatic, is important as it allows early intervention to target and address the risk factors to prevent further decline. In support of this, a recent review concluded that targeted CGA results in greater likelihood of independent living, better long-term cognitive outcomes, lower rates of institutional care and lower mortality, compared to general medical assessment (24). 


\section{References}

1. Frewen J, Finucane C, Cronin H, Rice C, Kearney PM, Harbison J, et al. Factors that influence awareness and treatment of atrial fibrillation in older adults. Qjm-Int J Med. 2013;106(5):415-24.

2. Leahy $\mathrm{S}, \mathrm{AM} \mathrm{OH}, \mathrm{N} O L$, Healy M, McCormack M, Kenny RA, et al. Prevalence and correlates of diagnosed and undiagnosed type 2 diabetes mellitus and pre-diabetes in older adults: Findings from the Irish Longitudinal Study on Ageing (TILDA). Diabetes Res Clin Pract. 2015;110(3):241-9.

3. Murphy CM, Kearney PM, Shelley EB, Fahey T, Dooley C, Kenny RA. Hypertension prevalence, awareness, treatment and control in the over 50s in Ireland: evidence from The Irish Longitudinal Study on Ageing. J Public Health (Oxf). 2016;38(3):450-8.

4. Cronin H, O'Regan C, Kenny R. Fifty Plus in Ireland 2011: First Results from The Irish Longitudinal Study on Ageing : Physical And Behavioural Health Of Older Irish Adults. Dublin: The Irish Longitudinal Study on Ageing; 2011.

5. O'Regan C, Cronin H, Kenny R. Fifty Plus in Ireland 2011: First Results from The Irish Longitudinal Study on Ageing: Mental Health And Cognitive Function Dublin: The Irish Longitudinal Study on Ageing; 2011.

6. Lara J, Cooper R, Nissan J, Ginty AT, Khaw KT, Deary IJ, et al. A proposed panel of biomarkers of healthy ageing. BMC Med. 2015;13:222.

7. Cooper R, Kuh D, Cooper C, Gale CR, Lawlor DA, Matthews F, et al. Objective measures of physical capability and subsequent health: a systematic review. Age Ageing. 2011;40(1):14-23.

8. Cooper R, Kuh D, Hardy R, Mortality Review G, Falcon, Teams HAS. Objectively measured physical capability levels and mortality: systematic review and metaanalysis. BMJ. 2010;341:c4467.

9. Podsiadlo D, Richardson S. The timed "Up \& Go": a test of basic functional mobility for frail elderly persons. J Am Geriatr Soc. 1991;39(2):142-8. 
10. Hypertension EETFftMoA. 2013 Practice guidelines for the management of arterial hypertension of the European Society of Hypertension (ESH) and the European Society of Cardiology (ESC): ESH/ESC Task Force for the Management of Arterial Hypertension. J Hypertens. 2013;31(10):1925-38.

11. Laurent S, Boutouyrie P, Asmar R, Gautier I, Laloux B, Guize L, et al. Aortic stiffness is an independent predictor of all-cause and cardiovascular mortality in hypertensive patients. Hypertension. 2001;37(5):1236-41.

12. Graham I, Atar D, Borch-Johnsen K, Boysen G, Burell G, Cifkova R, et al. European guidelines on cardiovascular disease prevention in clinical practice: executive summary. Fourth Joint Task Force of the European Society of Cardiology and other societies on cardiovascular disease prevention in clinical practice (constituted by representatives of nine societies and by invited experts). Eur J Cardiovasc Prev Rehabil. 2007;14 Suppl 2:E1-40.

13. National Cholesterol Education Program Expert Panel on Detection E, Treatment of High Blood Cholesterol in A. Third Report of the National Cholesterol Education Program (NCEP) Expert Panel on Detection, Evaluation, and Treatment of High Blood Cholesterol in Adults (Adult Treatment Panel III) final report. Circulation. 2002;106(25):3143-421.

14. Villareal DT, Apovian CM, Kushner RF, Klein S, American Society for N, Naaso TOS. Obesity in older adults: technical review and position statement of the American Society for Nutrition and NAASO, The Obesity Society. Obes Res. 2005;13(11):184963.

15. Harada CN, Natelson Love MC, Triebel KL. Normal cognitive aging. Clin Geriatr Med. 2013;29(4):737-52.

16. Colenbrander A. Visual standards, aspects and range of vision loss with emphasis on population surveys. Report prepared for the International Council of Ophthalmology at the 29th International Congress of Ophthalmology. Sydney; 2002.

17. Schott AM, Weillengerer S, Hans D, Duboeuf F, Delmas PD, Meunier PJ. Ultrasound Discriminates Patients with Hip Fracture Equally Well as Dual-Energy X-Ray Absorptiometry and Independently of Bone-Mineral Density. J Bone Miner Res. 1995;10(2):243-9. 
18. Varenna M, Sinigaglia L, Adami S, Giannini S, Isaia G, Maggi S, et al. Association of quantitative heel ultrasound with history of osteoporotic fractures in elderly men: the ESOPO study. Osteoporos Int. 2005;16(12):1749-54.

19. Kenny RA, Coen RF, Frewen J, Donoghue OA, Cronin H, Savva GM. Normative values of cognitive and physical function in older adults: findings from the Irish Longitudinal Study on Ageing. J Am Geriatr Soc. 2013;61 Suppl 2:S279-90.

20. Finucane C, O'Connell MDL, Fan CW, Savva GM, Soraghan CJ, Nolan H, et al. Age-Related Normative Changes in Phasic Orthostatic Blood Pressure in a Large Population Study Findings From The Irish Longitudinal Study on Ageing (TILDA). Circulation. 2014;130(20):1780-9.

21. Leahy S, Nolan A, O'Connell J, Kenny R. Obesity in an Ageing Society Implications for health, physical function and health service utilisation. Dublin: The Irish Longitudinal Study on Ageing; 2014.

22. Donoghue O, O'Connell M, Kenny R. Walking to Wellbeing: Physical Activity, Social Participation and Psychological Health in Irish adults aged 50 years and Older. Dublin: The Irish Longitudinal Study on Ageing; 2016.

23. National Clinical Programme for Older People. Comprehensive Geriatric Assessment, 2016. Available from: https://www.hse.ie/eng/about/Who/clinical/natclinprog/ olderpeopleprogramme/resources/ComprehensiveGeriatricAssessmentSummary.pdf.

24. Ellis G, Whitehead MA, O'Neill D, Langhorne P, Robinson D. Comprehensive geriatric assessment for older adults admitted to hospital. Cochrane Database Syst Rev. 2011(7):CD006211. 
